\title{
Validación de Alstroemeria parvula Phil. (Alstroemeriaceae)
}

\section{Validation of Alstroemeria parvula Phil. (Alstroemeriaceae)}

\author{
Mélica Muñoz-Schick ${ }^{1}$, Vanezza Morales² \& Andrés MoreiRa-Muñoz ${ }^{2}$ \\ ${ }^{1}$ Curadora emérita, Museo Nacional de Historia Natural, Casilla 787, Santiago, Chile. \\ ${ }^{2}$ Instituto de Geografía, Pontificia Universidad Católica de Chile, Vicuña Mackenna 4860, Macul, Santiago, Chile. \\ mmunoz@mnhn.cl
}

\begin{abstract}
Alstroemeria parvula Phil., is validated according to new recollections and observations in the Central Chilean Andes. It is redescribed and new localities are included confirming that it is solely protected in the private reserve and RAMSAR Site "Parque Andino Juncal”.
\end{abstract}

Alstroemeria parvula Phil. fue descrita por R.A.Philippi (1864) sobre la base de materiales recolectados por L. Landbeck, en los Andes de la provincia de Santiago (Las Arañas), y en Concumén [Cuncumén], provincia de Aconcagua (hoy provincia de Choapa, sur de la Región de Coquimbo). Se interpreta como Las Arañas el lugar de la recolección de Landbeck, ya que el total de los ejemplares de este colector en esa fecha corresponden a esta localidad.

Bayer (1987) la consideró como sinónimo dudoso de la especie Alstroemeria umbellata Meyen, ya que tampoco pudo encontrar material nuevo; sólo se encuentran los tipos, depositados en SGO, un ejemplar de Concumén [Cuncumén], y dos de Las Arañas. Hay duplicados de ellos en Ginebra (G) (dos ejemplares, según biblioteca virtual, JSTOR, www. plants.jstor.org). Tampoco fue considerada por MuñozSchick y Moreira-Muñoz (2003) pues a la fecha no se habían encontrado nuevos ejemplares en SGO, ni en CONC.

Durante una visita al Parque Andino Juncal en febrero 2008, nos encontramos en un sector con una Alstroemeria pequeña, casi pegada al suelo, pero en fruto. En una segunda excursión al lugar, a mediados de enero 2009, esta Alstroemeria crecía en forma abundante en el matorral andino. En una tercera excursión a fines de enero 2010, constatamos que la especie está presente en forma relativamente abundante en los $9 \mathrm{~km}$ de acceso al Parque (entre 2.200 y $2.300 \mathrm{msnm}$ ), así como en el interior del Parque (hasta ca. $2.600 \mathrm{msnm}$ ), aunque aparentemente en menor abundancia. La comparación del material recolectado con los ejemplares de SGO nos ha permitido llegar a la conclusión que se trata de la especie Alstroemeria parvula, descrita por R.A. Philippi.

\section{Alstroemeria parvula Phil.}

R.A. Philippi, Linnaea 33: 261. 1864.

TIPO: Región Metropolitana, provincia Santiago, Las Arañas,
I-1861, L. Landbeck (lectotipo, SGO 38136, designado por Bayer en 1987); Las Arañas, XII-1860, L. Landbeck (sintipo, SGO 38135). Región de Coquimbo, provincia Choapa (ex provincia de Aconcagua en el original), Concumén, I-1863, L. Landbeck (sintipo, SGO 38138).

\section{DESCRIPCIÓN}

Hierba perenne, de 6-12 cm de alto (hasta $15 \mathrm{~cm}$ ). Hojas basales linear-lanceoladas u obovadas hacia el ápice, nerviadas, de 5-7,5 cm de largo por 1-1,5 cm de ancho, glabras, bordes levemente ondulados y con escasas papilas, en su mayoría secas al momento de la floración, donde sólo quedan las tallinas que son linear-lanceoladas de 1,0-2,5 (-6) cm de largo por 2-4 (-5) $\mathrm{mm}$ de ancho. Inflorescencia de 3-7 rayos unifloros, pedúnculos de 1-1,5 cm de largo, tépalos de color rosado pálido, raro blancos (Fig. 1 A), de bordes denticulados; los externos obovado redondeados, de 2-2,5 cm de largo por 1,4 cm de ancho, adelgazados hacia la base, con mucrón corto, grueso, oscuro, prolongándose este color por el envés, los internos superiores de $2 \mathrm{~cm}$ de largo por 0,6 cm de ancho, más angostos, linear-oblongos, recurvos, ápice igualmente engrosado y oscuro, en la mitad más o menos con una franja amarillo-anaranjada, con manchitas purpúreas, que se prolongan hacia la base; tépalo inferior más ancho, algo romboideo, sin manchas ni franja y en él reposan los estambres de anteras oscuras y el estigma (Fig. 1 B). Cápsula de 1,6-1,7 cm de largo por 0,9-1 cm de ancho, color verde con costillas purpúreas, maduras son de color café; semillas redondeadas, color café, de 3 mm de diámetro, verrucosas.

Plantas que crecen en forma generalmente solitaria (Fig. $1 \mathrm{C})$. Florece entre diciembre y enero; frutos a fines de enero $\mathrm{y}$ en febrero. 
Clave para las especies de Alstroemeria presentes en la cordillera andina de las Regiones de Valparaíso y Metropolitana

1. Flores con los tépalos superiores internos muy recurvos

2. Hojas lineares, retorcidas, plantas hasta $1 \mathrm{~m}$ de alto.

A. revoluta

2'. Hojas linear-lanceoladas a obovado-redondeadas, no retorcidas, plantas de hasta $30 \mathrm{~cm}$ de alto.

3. Hojas basales obovado-redondeadas, arrosetadas y presentes al momento de la floración.

A. umbellata

3'. Hojas basales linear-lanceoladas a obovadas, no arrosetadas y generalmente secas al momento de la floración.

A. parvula

1’. Flores con tépalos superiores internos erectos o algo recurvos.

4. Flores con tépalos internos superiores sin mancha amarilla.

A. spathulata

4'. Flores con tépalos internos superiores con mancha amarilla

5. Mancha amarilla en una franja en la mitad de los tépalos.

A. pallida

5’. Mancha amarilla en los 2/3 inferiores de los tépalos.

A. exerens
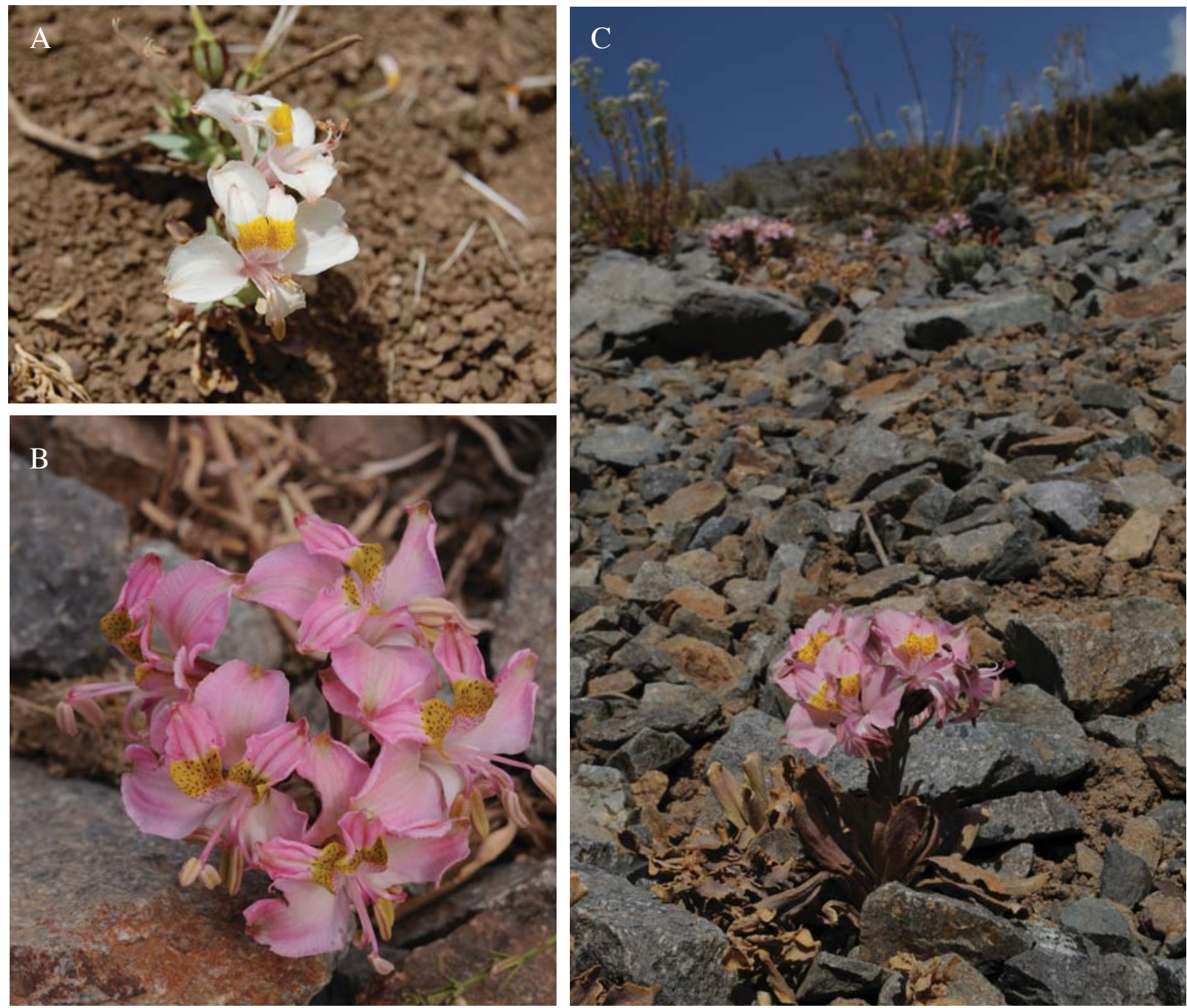

Figura 1. A. Alstroemeria parvula, de flores blancas (fotografía de F. Casado), B. detalle de flores rosadas (fotografía de A. MoreiraMuñoz), C. planta en su hábitat (fotografía A. Moreira-Muñoz).

Figure 1. Alstroemeria parvula, white flowered (photograph by F. Casado), B. rose flower details (photograph by A. Moreira-Muñoz), C. habitat of the plant (photograph by A. Moreira-Muñoz). 
DiSTRIBUCIÓN Y OBSERVACIONES DEL HÁBITAT

Es una especie cordillerana que habita desde el sur de las regiones de Coquimbo a la Metropolitana, entre los 2.2002.600 m s.n.m.

En la zona de ingreso al Parque Andino Juncal y en el Parque mismo crece en asociación con Chuquiraga oppositifolia D.Don, Nardophyllum lanatum (Meyen) Cabrera, Mutisia sinuata Cav., M. subulata Ruiz \& Pav., Rhodophiala rhodolirion (Baker) Traub; en los márgenes de la ruta internacional se encontró creciendo junto con algunos grupos de Alstroemeria spathulata C.Presl. En la cordillera de Quilapilún la especie ha sido encontrada en un depósito aluvial creciendo junto a Chuquiraga oppositifolia y Adesmia exilis Clos (ejemplar recolectado por García y Faúndez 725, SGO 153790).

El piso vegetacional corresponde al "matorral andino" (Muñoz-Schick et al. 2000), denominado por Luebert \& Pliscoff (2006) como "Matorral bajo mediterráneo andino de Chuquiraga oppositifolia y Nardophyllum lanatum”. Este piso vegetacional tiene una distribución en laderas altas de la cordillera de los Andes, desde el sur de la Región de Coquimbo hasta la Región de O’Higgins y se encuentra protegido en apenas un 2,4\% de su superficie (Luebert \& Pliscoff 2006). Hasta el momento sólo se ha reportado la presencia de poblaciones de A. parvula en el área protegida privada y sitio RAMSAR "Parque Andino Juncal”, único sitio de protección de la flora andina de la cuenca del Río Aconcagua.

\section{Material estudiado}

Región de Valparaíso, provincia Los Andes, primeras curvas hacia Portillo, 11-I-2007, M.T. Eyzaguirre \& P. Fürstenberg (SGO 154426); Entrada a Juncal, 3251'55” S; 708'48”W, 2.257 m s.n.m., 1-II-2008, A. Moreira 1053 (SGO 157438); Juncal, 3252'35” S; 708’12’W, 2.315 m s.n.m., 1-II-2008, A. Moreira 1059 (SGO 157444); Parque Andino Juncal, 6 $\mathrm{km}$ desde cruce ruta internacional al puente, $32^{\circ} 54^{\prime} 11,2$ ” S; 70 6’30,2” W, 2.384 m s.n.m., 9-II-2009, M. Muñoz 5074 (SGO 158764); 1-2 km entrando a Juncal, 3252’22,7” S;
708’40,2” W, 2.246 m s.n.m., 14-I-2010, A. Moreira, M. Muñoz \& V. Morales 1266 (SGO 159152); Bajada desde Portillo a Juncal, en la segunda curva desde abajo, 3251'55,8” S; 708'47,3” W, 2.255 m s.n.m., 14-I-2010, A. Moreira, M. Muñoz \& V. Morales 1263 (SGO 159149); Parque Andino Juncal, sendero a las Canchitas, sobre el Refugio, 3255’16,7” S; 705’7,5” W, 2.637 m s.n.m., 14I-2010, A. Moreira, M. Muñoz \& V. Morales 1279 (SGO 159061); Región Metropolitana, Prov. Chacabuco, cordillera de Quilapilún, loma La Leonera, 336’ S; 70³2’ W, 2.350 m s.n.m., 30-XII-2004, N. García \& L. Faúndez 725 (SGO 153790).

\section{AGRADECIMIENTOS}

A Catherine Kenrick, creadora e incansable impulsora del Parque Andino Juncal. A Francisco Casado y María Castro por el apoyo en terreno y las fotografías de la especie. Trabajo de campo financiado por proyecto FONDECYT Iniciación 11085016 (2008).

\section{BIBLIOGRAFÍA}

BAYer, E. 1987. Die Gattung Alstroemeria in Chile. Mitteilungen aus der Botanischen Staatssammlung München 24: 1-362.

Luebert, F. \& P. Pliscoff. 2006. Sinopsis bioclimática y vegetacional de Chile. Editorial Universitaria, Santiago, Chile. 307 pp.

Muñoz-Schick, M. \& A. Moreira-Muñoz. 2003. Alstroemerias de Chile: diversidad, distribución y conservación. Taller La Era, Santiago, 139 pp.

Muñoz-Schick, M., A. Moreira-Muñoz, C. Villlagrán \& F. Luebert. 2000. Caracterización florística y pisos de vegetación en los Andes de Santiago, Chile central. Boletín del Museo de Historia Natural, Chile, 49: 9-50.

Philippi, R.A. 1864. Plantarum novarum chilensium. Centuriae inclusis quibusdam Mendocinis et Patagonicis. Linnaea 33: 1-308.

Recibido: 28.09.10

Aceptado: 10.01.11 